\title{
Location Based Services: Research Trends and Open Challenges
}

\author{
Haosheng Huang a, *, Georg Gartner ${ }^{\text {b }}$, Jukka Krisp ${ }^{\text {c }}$, Martin Raubal ${ }^{\text {d }}$, Nico Van de Weghe ${ }^{\text {e }}$ \\ ${ }^{a}$ GIScience Center, Department of Geography, University of Zurich, Zurich, Switzerland, haosheng.huang@geo.uzh.ch \\ ${ }^{b}$ Research Group Cartography, Department of Geodesy and Geoinformation, TU Wien, Vienna, Austria, \\ georg.gartner@tuwien.ac.at \\ ${ }^{c}$ Research Group Applied Geoinformatics, University of Augsburg, Augsburg, Germany, jukka.krisp@ geo.uni-augsburg.de \\ ${ }^{d}$ Institute of Cartography and Geoinformation, ETH Zurich, Zurich, Switzerland, mraubal@ethz.ch \\ ${ }^{e}$ Department of Geography, Ghent University, Ghent, Belgium, Nico.VandeWeghe@UGent.be \\ * Corresponding author
}

Keywords: Location Based Services, Research Trends, Research Agenda, Context-aware Computing

\begin{abstract}
:
We are now living in a mobile information era, which is fundamentally changing science and society. Location Based Services (LBS), which deliver information depending on the location of the (mobile) device and user, play a key role in this mobile information era.

This presentation will review the state-of-the-art in LBS research, and identify several research trends in recent years. These range from mobile guides and navigation systems to more diverse applications, from outdoor to indoor and mixed outdoor/indoor environments, from location based to context-aware, from maps and audio to more diverse and 'natural' interfaces, from technology-oriented to interdisciplinary research, and analysis of big spatial data.

To motivate further LBS research and stimulate collective efforts, this presentation will present a series of key research challenges that are essential to advance the development of LBS, setting a research agenda for LBS to 'positively' shape the future of our mobile information society. These research challenges (Figure 1) cover issues related to the core of LBS development (e.g. positioning, modelling, and communication), evaluation, and analysis of LBS-generated data, as well as social, ethical, and behavioural issues that rise as LBS enter into people's daily lives. This research agenda is an outcome of the multi-phase collaborative initiative (https://lbs.icaci.org/research-agenda/), started in May 2016.
\end{abstract}

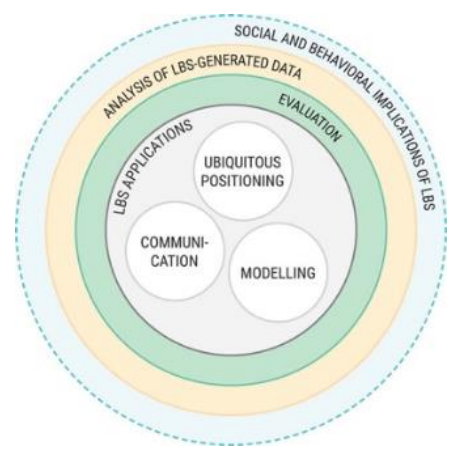

Figure 1. The 'key research challenges' organised into seven broad areas: positioning, modelling, communication, evaluation, applications, analysis of LBS-generated data, social and behavioural implications of LBS.

We hope this research agenda helps to motivate further LBS research and stimulate collective efforts. To bring together the broader community to answer these open research questions, a series of activities (conferences, workshops, and special issues) will be organized in the coming years. By working collaboratively within the LBS community and across cognate communities, we can ensure a better future of our mobile information society.

This presentation is based on the following publication: Haosheng Huang, Georg Gartner, Jukka M. Krisp, Martin Raubal \& Nico Van de Weghe (2018): Location based services: ongoing evolution and research agenda, Journal of Location Based Services, DOI: 10.1080/17489725.2018.1508763. 